\title{
Reducing long term sickness absence by an activating intervention in adjustment disorders: a cluster randomised controlled design
}

\author{
J J L van der Klink, R W B Blonk, A H Schene, F J H van Dijk
}

Occup Environ Med 2003;60:429-437

See end of article for authors' affiliations

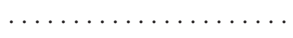

Correspondence to: Dr J J L van der Klink, Academic Medical Center, Coronel Institute, PO 22660, 1100 DD Amsterdam, Netherlands. i.j.vanderklink@amc.uva.nl

Accepted

11 October 2002
Aims: To compare an innovative activating intervention with "care as usual" (control group) for the guidance of employees on sickness leave because of an adjustment disorder. It was hypothesised that the intervention would be more effective than care as usual in lowering the intensity of symptoms, increasing psychological resources, and decreasing sickness leave duration.

Methods: A prospective, cluster randomised controlled trial was carried out with 192 patients on first sickness leave for an adjustment disorder. Symptom intensity, sickness duration, and return to work rates were measured at 3 months and 12 months. Analyses were performed on an intention to treat basis.

Results: At 3 months, significantly more patients in the intervention group had returned to work compared with the control group. At 12 months all patients had returned to work, but sickness leave was shorter in the intervention group than in the control group. The recurrence rate was lower in the intervention group. There were no differences between the two study groups with regard to the decrease of symptoms. At baseline, symptom intensity was higher in the patients than in a normal reference population, but decreased over time in a similar manner in both groups to approximately normal levels.

Conclusion: The experimental intervention for adjustment disorders was successful in shortening sick leave duration, mainly by decreasing long term absenteeism.
S ickness leave is generally considered as a major source of societal costs in Western countries. With the increase in mental workload of the past decades, the fraction of psychological problems related to occupational stress has increased rapidly. These problems are also reported in the literature as emotional distress or stress related disorders. For example, the United Nations International Labour Organisation $^{1}$ estimated the cost of work absence and loss of productivity at US $\$ 200$ billion a year for the USA. Comparable figures have been reported for European countries. ${ }^{2}$ Nevertheless, little is known about the efficacy and effectiveness of interventions used for patients with emotional distress. ${ }^{34}$

If emotional distress goes together with sickness leave, it concurs with the DSM IV classification "adjustment disorder". ${ }^{5}$ Although adjustment disorders can be considered as minor psychiatric morbidity, Schröer reported that at least $20 \%$ of patients with such a disorder do not return to work within a year, usually leading to loss of employment. ${ }^{6}$ Adjustment disorders account for most psychopathology giving rise to inability to work in the Netherlands, whereas psychiatric illnesses, such as major depression, anxiety disorders, psychoses, and personality disorders, account for only a small minority of cases. ${ }^{7}$ Indeed, in the Netherlands, $33 \%$ of disability benefit payments for chronic illness are for mental disorders. At present, more than $50 \%$ of these are stress related disorders such as adjustment disorders. Despite the high prevalence of disabling adjustment disorders, there has been relatively little research on treatment efficacy in the occupational health care setting, and thus an effective treatment strategy has yet to be determined.

In a recent meta-analysis we found that, in a preventive context, cognitive-behavioural interventions were more effective than other interventions. ${ }^{4}$ Such interventions had most effect on psychopathological symptoms and on psychological resources and responses (for example, mastery); they also had a small but significant effect on perceived quality of work life. They did not appear to affect work ability.

As the main goal of the present study was to evaluate an intervention aimed at preventing the disabling long term consequences of adjustment disorders, we looked for interventions effective on work ability and sickness leave duration outside the traditional domain of psychopathology. Positive results on these outcome measures have been reported from time contingent approaches in low back pain patients. ${ }^{89} \mathrm{~A}$ time contingent approach implies that activities increase according to a prestructured time scheme. Thus, the building up is not dependent on the course of symptoms. This avoids the pitfall of the sequence: "I can only start doing this or that when my fatigue (or any other symptom) has diminished", with the risk of more and more avoidance of activities and deterioration of symptoms.

On the basis of these findings, we developed and evaluated a new intervention for adjustment disorders, based on the principles of time contingency and cognitive behavioural treatment. The main aim of the intervention was to activate patients to develop and implement problem solving strategies for daily (working) life problems. Problem solving activities were planned and built up in time according to a time contingent scheme. In the present study we use "care as usual", as the control group. The outcomes studied were: symptoms, mastery, and sickness leave duration.

\section{METHODS}

Setting

The present study was conducted at Royal KPN (Royal PTT Nederland). At the time of the study, the (private) company

Abbreviations: $4 D S Q$, Four-Dimensional Symptom Questionnaire DWHQ, Dutch Work and Health Questionnaire; OP, occupational physician; SCL-90, Symptom Checklist-90; UCL, Utrecht Coping List 
comprised both Postal and Telecom Services and had approximately 100000 employees. The company had well defined human resource policies. The in-company occupational health service cooperated with company management in the prevention and management of health problems and absenteeism. Occupational physicians were geographically spread over the country and served fixed company divisions and employee populations. In the years preceding the study, several courses had been organised for occupational physicians to improve the recognition, diagnosis, and treatment of mental health problems. Company return to work rates were considerably more favourable than in the general Dutch situation. Nevertheless, the occupational health service management felt the need for an occupational physician training programme aimed at a more structured and defined intervention on employees with occupational stress.

\section{Design, selection, and randomisation procedure}

The study is a cluster randomised trial with an intervention group and a control (care as usual) group. Clustering was on the occupational physician level. Self report measures were used at baseline and at 12 and 52 weeks. Duration of sickness leave and incidence of recurrence were measured over a one year period.

Almost all occupational physicians of the occupational health service (response: 96\%) volunteered after an invitation to participate in the study. Patient randomisation is preferable but was not possible in this study, because of company policies. The company did not accept an interference of the fixed relation between patients and occupational physicians. Therefore the physicians were randomised into two groups: 17 occupational physicians (OPs) were assigned to the intervention group and 16 to the control group. The mean number of patients included by the OPs was six, varying from one to 16 . Occupational physicians were stratified by experience and number of years working for the company because these aspects might have influence on the quality of contacts with management and therapists. Quality of these contacts may have an influence on the effectiveness of the intervention. An OP was considered to be experienced when he or she had five or more years OP experience, of which at least two were in service of the company.

The randomisation was conducted blindly by an independent research assistant who assigned two groups of occupational physicians (experienced and not experienced) to the two study groups.

All occupational physicians were instructed on how to inform patients about the study. Informed consent was obtained after patients had received information about the primary purpose of the trial. Both occupational health strategies were presented in a global way and as equally effective to the participants. Patients were not aware of which treatment they received. Patients were asked to fill in questionnaires at baseline, 3 months, and 12 months. Patients were included from May 1995 to July 1996.

\section{Subjects}

All employees who were two weeks on sick leave were referred to their occupational physician. To be included, patients had to be on their first sickness leave because of an adjustment disorder. A checklist with inclusion and exclusion criteria was used. The checklist was construed in such a way that all included patients met the DSM IV criteria for adjustment disorder. For inclusion, there had to be a recent ( $<3$ months) identifiable psychosocial stressor similar to that defined in the DSM IV ${ }^{5}$ and the patient had to have at least eight of 17 distress symptoms representing the main symptom categories of the DSM IV adjustment disorder. ${ }^{5}$ Patients were excluded if one of the DSM IV exclusion criteria for an adjustment disorder held (for example, the patient had a depression), ${ }^{5}$ if there had been a period of guidance for an adjustment disorder in the preceding year, if there was physical comorbidity that could have an effect on absenteeism, if communication in Dutch was not possible, if the patient was pregnant or had a recent delivery ( $<6$ months), and if employment at KPN would terminate within six months.

Patients who met the inclusion criteria and filled in the baseline questionnaire entered the trial according to the treatment group their occupational physician was assigned to.

\section{Treatment}

\section{Intervention}

The importance of an early start of the intervention aimed at acquisition of coping skills and at regaining control was emphasised to the occupational physicians in the intervention group. The intervention comprised a graded activity approach and was based on a three stage model, resembling stress inoculation training, ${ }^{10}{ }^{11}$ a highly effective form of cognitive behavioural treatment. ${ }^{12}$ In the first stage, there was emphasis on information: understanding the origin and cause of the loss of control. Patients were also stimulated to do more nondemanding daily activities. In the second stage, patients were asked to draw up an inventory of stressors and to develop problem solving strategies for these causes of stress. ${ }^{13}$ In the third stage, patients put these problem solving strategies into practice and extend their activities to include more demanding ones. The patients' own responsibility and active role in the recovery process was emphasised.

Occupational physicians in the intervention group underwent a three day training course given by an experienced occupational physician/psychologist, a psychologist/therapist, an experienced general practitioner/researcher on emotional distress, and a psychiatrist. They were trained in multiple cognitive-behavioural, prescriptive interventions to stimulate the patients' acquisition of problem solving skills, and to structure the patients' daily activities. The occupational physicians were free to choose the specific tools they used in each phase of the process. Specified and prescribed in a protocol were aspects concerning the procedure of the intervention: the OPs had to plan four or five consultations in the first six weeks of sickness leave with a total length over these sessions of at least 90 minutes. All sessions were based on an individual doctor-patient consultation with the OP. At least three contacts with company management were prescribed in the first three months. For the intervention group at least one session was prescribed after work resumption, focused on relapse prevention.

To increase treatment integrity, the use of tools was supervised by those responsible for the training. The occupational physicians filled in forms to record their activities.

\section{Care as usual}

There was neither a professional nor a company guideline for the care of patients with adjustment disorders. As there have been several courses on mental health problems in the preceding years there was a shared concept of diagnosis and guidance. The occupational physicians in this group were aware of the three stage model, but most had not been trained in its use and did not structure their guidance according to it. In general, "usual" care was based on empathic counselling, instruction about stress, lifestyle advice, and discussion of work problems with the patient and company management.

Occupational physicians in this group received no training in guidance. However, they received a three hour session on the use of the inclusion and exclusion criteria and on how to record their guidance activities relevant for this study.

\section{Measures and time scheme}

Information about sociodemographic characteristics, quality of work life, and coping style were obtained from the baseline 


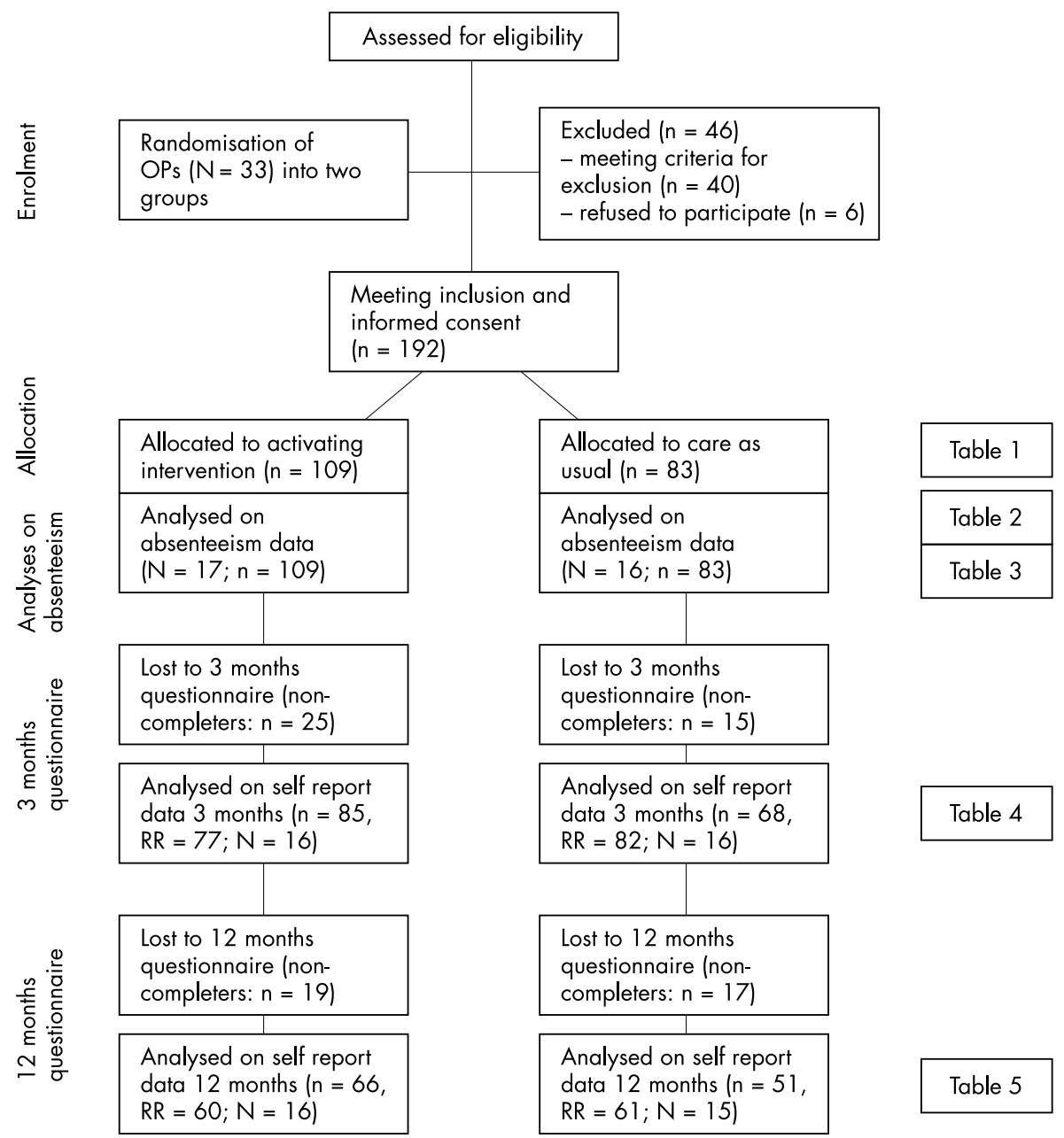

Figure 1 Flow diagram of subjects' progress. $n=$ number of patients, $N=$ number of $\mathrm{OPs}, \mathrm{RR}=$ overall response rate. questionnaire. Consistent with the meta-analysis ${ }^{4}$ and with our main goal, we assessed symptoms, "mastery" to cover "psychological resources and responses", and absenteeism at baseline, 3 months, and 12 months. All self report measures used had Cronbach alphas higher than the 0.70 criterion formulated by Nunnaly. ${ }^{14}$

The baseline questionnaire was given at the end of the first consultation to the patients by their OP with a reply envelope for the independent research institute where the data were administered. Reminders and the 3 and 12 months questionnaires were sent to the patients from the research institute. Research assistants who administered the questionnaires had no knowledge of which study group responders belonged to.

\section{Baseline measures}

The Dutch Work and Health Questionnaire (DWHQ)

This validated questionnaire ${ }^{15}$ assesses the appraisal of employees of the quality of their work and health. It consists of eight scales assessing work appreciation and stressors and two scales on health. We used the eight scales on work (32 items) for a combined total score of quality of work life. The two scales on health were omitted because of redundancy with other measures.

\section{Utrecht Coping List (UCL)}

This validated 47 item inventory, ${ }^{16}{ }^{17}$ subdivided in seven scales, measures coping from a dispositional point of view, conceptualising coping as a personality characteristic. A four point Likert scale is used for each item. We used the total score to assess overall coping skills. In addition, we applied the "active problem solving" scale as acquisition of coping skills, especially active coping skills, was an important goal of the intervention.

\section{Outcome measures}

Four-Dimensional Symptom Questionnaire (4DSQ)

The $4 \mathrm{DSQ}^{18}$ was developed to assess psychopathology among patients attending general practitioners. The questionnaire comprises 50 items devided over four scales measuring distress, depression, anxiety, and physical symptoms. The 4DSQ was developed and validated for primary health care patients with adjustment disorders. ${ }^{19}$

\section{Symptom Checklist-90 items (SCL-90)}

The SCL- $90^{20} 21$ is a validated measure for psychopathological screening and useful for evaluation of treatment effects. The Dutch version, developed by Arrindell and Ettema, ${ }^{22} 23$ has a factorial structure closely related to the original instrument by Derogatis. ${ }^{20}$ To reduce the number of analyses the total score was used.

\section{Mastery Scale}

This scale assesses the extent to which a person regards life changes as being under his or her control in contrast to being ruled by fate. ${ }^{24}$ The scale consists of seven items, with a five point Likert scale for each item.

\section{Absenteeism}

Time to partial and to full return to work, duration of sickness leave, time to recurrence, incidence of recurrence in the year following full return to work, and prevalence rate of return to work at 3 and 12 months were used as effect parameters for 
Table 1 Baseline characteristics of intervention and control groups

\begin{tabular}{|c|c|c|c|}
\hline & \multicolumn{2}{|l|}{ Total $(n=192)$} & \multirow[b]{2}{*}{$\mathrm{p}$ value } \\
\hline & $\begin{array}{l}\text { Intervention } \\
\text { group }(n=109)\end{array}$ & $\begin{array}{l}\text { Control group } \\
(\mathrm{n}=83)\end{array}$ & \\
\hline \multicolumn{4}{|l|}{ Patient characteristics } \\
\hline Gender, \% male & 66 & 59 & 0.28 \\
\hline Age, mean (SD) & $39(8.0)$ & $42(8.8)$ & 0.02 \\
\hline Married state & & & 0.56 \\
\hline$\%$ married & 62 & 67 & \\
\hline$\%$ unmarried & 27 & 22 & \\
\hline$\%$ divorced & 11 & 11 & \\
\hline Highest level of education & & & 0.27 \\
\hline$\%$ elementary & 7 & 17 & \\
\hline$\%$ secondary & 70 & 66 & \\
\hline$\%$ higher & 12 & 4 & \\
\hline$\%$ other & 11 & 13 & \\
\hline \multicolumn{4}{|l|}{ Work related characteristics } \\
\hline Years of service at Royal PTT Nederland, $\%>10$ years & 62 & 76 & 0.15 \\
\hline In present job, $\%>5$ years & 41 & 51 & 0.35 \\
\hline$\%$ managerial position & 18 & 12 & 0.28 \\
\hline Hours of appointment, mean (SD) & $36(9.34)$ & $32(11.8)$ & 0.01 \\
\hline Quality of work life (DWHQ), mean (SD) & $1.42(0.19)$ & $1.38(0.21)$ & 0.24 \\
\hline \multicolumn{4}{|l|}{ Coping/control } \\
\hline Active problem solving (UCL), mean (SD) & $2.41(0.46)$ & $2.31(0.53)$ & 0.18 \\
\hline Total coping (UCL), mean (SD) & $2.13(0.25)$ & $2.11(0.26)$ & 0.71 \\
\hline \multicolumn{4}{|l|}{ Absenteeism } \\
\hline No. of spells in previous year, mean (SD) & $2.2(1.9)$ & $2.3(1.6)$ & 0.66 \\
\hline
\end{tabular}

absence from work. Time to return to work was defined as the period between the onset of sickness leave and first return to work, which was most of the time partial. Time to full return to work was defined as the period between the onset of sickness leave and full return to work. Duration of sickness leave was defined as the number of days lost until full return to work with a correction for partial return. Time to recurrence was defined as the period between the moment of full return to work and recurrence of sick leave for any reason, and the incidence of recurrence as the number of recurrences in a period of 12 months from full return to work. Absenteeism data were obtained from the company's computerised record system and were collected until one year after full return to work, with a maximum of two years after study entry.

\section{Statistical analysis}

The data in our study have a hierarchical, multilevel structure: OPs were randomised and trained for a guidance strategy that should show its effect on outcome (dependent) variables on the patients' level. Because of this, outcomes on the patients' level might be influenced by characteristics at the OP level and therefore be correlated at this level. ${ }^{25}{ }^{26}$ This may lead to an overestimation of significance when data are analysed on the individual level. With multilevel analysis data can be analysed in such a way that is accounted for the "hierarchical" structure of the data. We therefore conducted multilevel analyses where possible - that is, on the self report data at 3 and 12 months. The assumption of these techniques is that outcome variables are normally distributed. The outcome variables depression and anxiety are not normally distributed. However, because we corrected for baseline values the analyses actually deal with the changes in those variables. The changes are approximately normally distributed-that is, the residuals of the analyses performed were approximately normally distributed.

With respect to the absenteeism data we encountered two problems. Firstly, there are not yet techniques with a proven reliability in which survival analyses can be conducted in a multilevel structure. Survival analyses are considered as techniques of first choice for the analysis of absenteeism data.
Secondly, methods for analysing binary (dichotomous) outcome data on cluster level are not as well established as methods for analysing continuous outcome data. ${ }^{27}$ These data cannot be analysed reliably in a multilevel structure. The simplest approach to analyse binary data from a cluster randomisation trial is to obtain a single summary score for each cluster. Then the analyses can be conducted at the same level as the random assignment using standard statistical procedures. ${ }^{27}$ We also conducted the survival analyses on cluster level because of methodological rigour. We used the means for each cluster for these cluster level survival (time to event) analyses. In these analyses cluster size was introduced as weighting factor. As the point of inference in our study is so clearly on the individual level we also present outcomes of analyses on the individual level.

Analyses of variance (ANOVA) were conducted to check for systematic differences between included and excluded patients and between completers and drop outs during the study for intervention and control group. When significant differences were found in the baseline variables between intervention and control group, those variables were introduced as covariates in the analyses.

Kaplan-Meier survival analyses and Cox's proportional hazards regression analyses were used to analyse the absenteeism data. As terminal events in separate analyses, "time to return to work", "time to full return to work", and "time to recurrence" were used. Kaplan-Meier analyses were used to obtain means, medians, and confidence intervals. The statistical testing of significance was conducted with Cox regression analyses. These multivariate analyses made it possible to introduce significant differences in the baseline variables between intervention and control groups as covariates in the analyses.

Mann-Whitney U tests were conduced to analyse the rate of partial and of full return after three months and the incidence of recurrence in the year following full return to work.

Multilevel analyses were performed using MLwiN version 1.02.0002. The remaining analyses were performed using SPSS, version 9.0 (SPSS Inc., Illinois, USA). 
Table 2 Cluster level analyses (OP level; $n=33$ ) on absenteeism in intervention and control groups

\begin{tabular}{|c|c|c|c|}
\hline & \multicolumn{2}{|l|}{ Total $(n=33)$} & \multirow[b]{2}{*}{$\mathrm{p}$ value } \\
\hline & Intervention group $(n=17)$ & Control group $(n=16)$ & \\
\hline \multicolumn{4}{|l|}{ Return to work rates } \\
\hline$\%$ return to work (partial or full) at 3 months & $98 \%$ & $87 \%$ & 0.01 \\
\hline$\%$ full return to work at 3 months & $79 \%$ & $64 \%$ & 0.08 \\
\hline$\%$ full return to work at 12 months & $100 \%$ & $100 \%$ & - \\
\hline \multicolumn{4}{|l|}{ Time to (full) return to work (days) } \\
\hline Median time to return to work $(95 \% \mathrm{Cl})$ & 37 (32 to 42 ) & $51 \quad(35$ to 67$)$ & \multirow{2}{*}{0.00} \\
\hline Mean time to return to work $(95 \% \mathrm{Cl})$ & $36(31$ to 40$)$ & 53 (44 to 62) & \\
\hline Median time to full return to work $(95 \% \mathrm{Cl})$ & $60(52$ to 67$)$ & 83 (79 to 88$)$ & \multirow{2}{*}{0.10} \\
\hline Mean time to full return to work $(95 \% \mathrm{CI})$ & 67 (51 to 83$)$ & 94 (71 to 117$)$ & \\
\hline \multicolumn{4}{|l|}{ Duration of sickness leave (days) } \\
\hline Median duration of sickness leave $(95 \% \mathrm{Cl})$ & $46(41$ to 51$)$ & $67(40$ to 94$)$ & \multirow{2}{*}{0.02} \\
\hline Mean duration of sickness leave $(95 \% \mathrm{CI})$ & $49(40$ to 58$)$ & 73 (55 to 92$)$ & \\
\hline \multicolumn{4}{|l|}{ Recurrence } \\
\hline $\begin{array}{l}\text { Mean incidence (no. of spells) of recurrence in a one year } \\
\text { period after full return to work }\end{array}$ & 1.9 & 2.2 & 0.26 \\
\hline Median time to recurrence leave $(95 \% \mathrm{Cl})$ & 181 (156 to 206$)$ & 162 (148 to 177$)$ & \multirow{2}{*}{0.54} \\
\hline Mean time to recurrence leave $(95 \% \mathrm{CI})$ & $187(158$ to 216$)$ & 179 (156 to 202) & \\
\hline
\end{tabular}

Time to return to work: period between onset of sickness leave and first return to work (most of the time partial).

Time to full return to work: period between onset of sickness leave and full return to work.

Duration of sickness leave: number of days lost until full return to work with a correction for partial return.

Time to recurrence: period between full return to work and recurrence of sick leave for any reason.

Incidence of recurrence: number of recurrences in a period until 12 months from full return to work.

$p$ value from multivariate (Cox) analyses with cluster size as covariate.

\section{RESULTS}

\section{Subjects}

A total of 238 patients were eligible for the study because they had one or more symptoms of emotional distress (fig 1). Six refused to participate in the study and 40 met the exclusion criteria. Thus 192 patients met the inclusion criteria and gave their informed consent. Of these, $153(79 \%)$ responded to the second questionnaire at 3 months. A total of 117 (61\%) patients responded to the 12 months questionnaire. This implies a dropout percentage of 39\% by 12 months for the self report analyses. Absenteeism analyses could be performed for all included patients $(n=192)$.

\section{Comparison of pretest scores, randomisation}

A total of 109 patients comprised the intervention group and 83 the control group. There were no significant differences between the two groups in symptoms reported on the checklist for inclusion or exclusion.

Analysis of sociodemographic measures showed that the patients in the intervention group were significantly younger than their counterparts in the control group, and that they worked more hours a week (both $\mathrm{p}<0.05$ ). Therefore, we introduced "age" and "hours of appointment" as covariates in the analyses. Table 1 shows the baseline characteristics of the two groups. At baseline, the two groups did not differ significantly on the outcome measures (4DSQ, SCL-90 total score, and Mastery Scale).

Compared with a psychiatric outpatients reference population, both groups scored below average on psychopathology (SCL-90 total), corresponding to a more healthy status. Compared with a healthy reference population, both groups scored above the 80th centile. We used reference values provided in

Table 3 Patient level analyses $(n=192)$ on absenteeism in intervention and control groups

\begin{tabular}{|c|c|c|c|}
\hline & \multicolumn{2}{|l|}{ Total $(n=192)$} & \multirow[b]{2}{*}{$\mathrm{p}$ value } \\
\hline & Intervention group $(n=109)$ & Control group $(n=83)$ & \\
\hline \multicolumn{4}{|l|}{ Return to work rates } \\
\hline$\%$ return to work (partial or full) at 3 months & $97 \%$ & $86 \%$ & 0.00 \\
\hline$\%$ full return to work at 3 months & $78 \%$ & $63 \%$ & 0.02 \\
\hline$\%$ full return to work at 12 months & $100 \%$ & $100 \%$ & - \\
\hline \multicolumn{4}{|l|}{ Time to (full) return to work (days) } \\
\hline Median time to return to work $(95 \% \mathrm{Cl})$ & 33 (29 to 37$)$ & 38 (30 to 46$)$ & \multirow{2}{*}{0.00} \\
\hline Mean time to return to work $(95 \% \mathrm{Cl})$ & 36 (32 to 40$)$ & 53 (43 to 62) & \\
\hline Median time to full return to work $(95 \% \mathrm{Cl})$ & 47 (41 to 53$)$ & 63 (43 to 83$)$ & \multirow{2}{*}{0.03} \\
\hline Mean time to full return to work $(95 \% \mathrm{CI})$ & $69(58$ to 80$)$ & 91 (75 to 107$)$ & \\
\hline \multicolumn{4}{|l|}{ Duration of sickness leave (days) } \\
\hline Median duration of sickness leave $(95 \% \mathrm{CI})$ & 41 (35 to 46$)$ & $50(44$ to 56$)$ & \multirow{2}{*}{0.00} \\
\hline Mean duration of sickness leave $(95 \% \mathrm{Cl})$ & $49(43$ to 55$)$ & $70(58$ to 82$)$ & \\
\hline \multicolumn{4}{|l|}{ Recurrence } \\
\hline $\begin{array}{l}\text { Mean incidence (no. of spells) of recurrence in a one year } \\
\text { period after full return to work }\end{array}$ & 1.8 & 2.3 & 0.02 \\
\hline Median time to recurrence leave $(95 \% \mathrm{CI})$ & 186 (143 to 229$)$ & 170 (121 to 219$)$ & \multirow{2}{*}{0.24} \\
\hline Mean time to recurrence leave $(95 \% \mathrm{Cl})$ & $194(174$ to 213$)$ & 173 (152 to 195$)$ & \\
\hline
\end{tabular}

Time to return to work: period between onset of sickness leave and first return to work (most of the time partial).

Time to full return to work: period between onset of sickness leave and full return to work.

Duration of sickness leave: number of days lost until full return to work with a correction for partial return.

Time to recurrence: period between full return to work and recurrence of sick leave for any reason.

Incidence of recurrence: number of recurrences in a period until 12 months from full return to work.

$p$ value from multivariate (Cox) analyses with age and hours of appointment as covariates. 


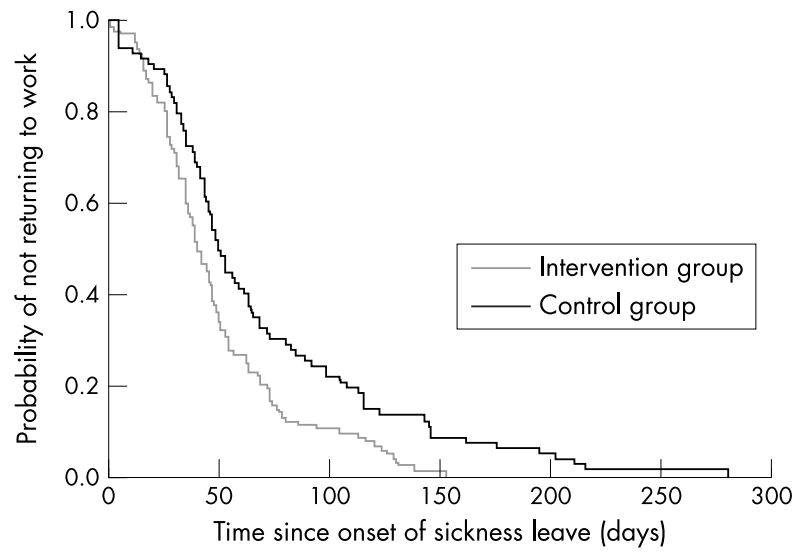

Figure 2 Cumulative probability of not returning to work since onset of sickness leave in invervention $(n=109)$ and control $(n=83)$ groups.

the Dutch SCL-90 manual ${ }^{23}$ converted to a population with $69 \%$ males and $31 \%$ females.

\section{Dropout analysis}

Analysis between completers on 3 months $(n=153)$ and dropouts after inclusion $(n=40)$ revealed that completers were older (mean age $41 v 37$ years, $\mathrm{p}<0.05$ ), worked more hours per week (mean $36 v 30$ hours, p $<0.05$ ), and had a lower mean SCL-90 total score (181 v 200, p < 0.05). There was no significant interaction with type of intervention.

Analysis between completers on 12 months $(n=117)$ and dropouts after inclusion $(n=76)$ revealed that there were more males among completers (68\% v 54\%, p < 0.05), that they worked more hours per week (mean $36 v 32$ hours, $\mathrm{p}<0.05$ ), and had a lower incidence of absenteeism periods in the year before the onset of this period (mean number of spells $2.0 v 2.7, \mathrm{p}<0.05)$. There was no significant interaction with type of intervention.

We finally conducted an analysis between completers on 12 months $(\mathrm{n}=117)$ and dropouts since the 3 months assessment $(\mathrm{n}=36)$. There was only one significant difference: completers had a lower incidence of absenteeism periods in the year before the onset of this period (mean number of spells $2.0 \vee 2.7, \mathrm{p}<0.05)$. This difference was greater for the intervention group ( $1.8 \vee 3.0$ periods of absenteeism) than for the control group ( $2.1 \vee 2.3$ absenteeism periods).

\section{Intervention}

Total contact duration was about 25 minutes longer in the intervention group compared with the control group over approximately five consultations for both groups, a significant difference. Ninety three per cent of the OPs in the intervention group applied at least one specific tool versus $20 \%$ of the OPs in the control group.

\section{Treatment outcomes \\ Absenteeism \\ Cluster level analyses}

The percentage of employees who returned to work within three months was significantly higher in the intervention group. For full return to work the difference was marginally significant (see table 2 ).

Time to return to work and duration of sickness leave were significantly shorter for the intervention group $(\mathrm{p}<0.05)$; for time to full return to work the difference was not significant $(\mathrm{p}=0.10)$ (table 2$)$. The rate ratios were 4.80 (95\% confidence interval (CI) 1.91 to 12.02 ) for time to return to work, 1.81 (95\% CI 0.89 to 3.65 ) for time to full return to work, and 2.39 (95\% CI 1.15 to 4.95 ) for duration of sickness leave.

\section{Patient level analyses}

The percentage of employees who returned to work within three months was significantly higher in the intervention group (see table 3 ). This was also the case for full return to work.

Time to return to work, time to full return to work, and duration of sickness leave were significantly shorter for the intervention group $(\mathrm{p}<0.05)$; table 3 shows means and medians. The rate ratios were 1.61 (95\% CI 1.18 to 2.19 ) for time to return to work and 1.41 (95\% CI 1.04 to 1.92 ) for time to full return to work, taking into account age and hours of appointment as covariates. All patients, irrespective of treatment group, had returned to work within 12 months. Figure 2 shows that the intervention predominantly shows an effect from about six weeks onwards. The mean incidence of recurrence in the one year period was lower for the intervention group $(\mathrm{p}=0.02)$. There was a non-significant difference of 20 days in mean time to recurrence.

\section{Self report}

Patients improved significantly on all outcomes within the first 3 months in both groups. However, there were no significant differences between the two groups (see table 4). At 3

Table 4 Health symptoms and mastery: baseline and 3 months

\begin{tabular}{|c|c|c|c|c|c|c|c|}
\hline & \multicolumn{4}{|c|}{ Total $(n=153)$} & \multicolumn{3}{|c|}{ Intervention effect } \\
\hline & \multicolumn{2}{|c|}{ Intervention group $(n=85)$} & \multicolumn{2}{|c|}{ Control group $(n=68)$} & \multicolumn{3}{|l|}{ Statistic } \\
\hline & Baseline & 3 months & Baseline & 3 months & $B_{1 \mathrm{i}}$ & $\mathrm{Cl}$ & $\mathrm{p}$ \\
\hline \multicolumn{8}{|l|}{ Symptoms } \\
\hline Distress, mean (SD) & $21.35(6.4)$ & $10.6(7.0)$ & $22.0(7.0)$ & $12.5(9.3)$ & 0.029 & -0.122 to 0.180 & 0.35 \\
\hline Depression, mean (SD) & $2.54(2.9)$ & $0.98(2.0)$ & $3.37(3.1)$ & $1.58(2.7)$ & 0.024 & -0.096 to 0.147 & 0.35 \\
\hline Anxiety, mean (SD) & $4.04(4.4)$ & $2.03(2.9)$ & $5.96(5.8)^{*}$ & $3.07(4.9)$ & -0.014 & -0.100 to 0.072 & 0.63 \\
\hline Physical symptoms, mean (SD) & $12.37(6.1)$ & $7.41(5.4)$ & $12.8(5.8)$ & $8.06(5.7)$ & 0.003 & -0.089 to 0.095 & 0.50 \\
\hline \multicolumn{8}{|l|}{ SCL-90 } \\
\hline Total score, mean (SD) & $176(43)$ & $133(34)$ & $188(48)$ & $142(49)$ & -0.025 & -0.174 to 0.124 & 0.63 \\
\hline \multicolumn{8}{|l|}{$\begin{array}{l}\text { Psychological resources and } \\
\text { responses }\end{array}$} \\
\hline Mastery, mean (SD) & $3.24(0.63)$ & $3.56(0.68)$ & $3.18(0.65)$ & $3.49(0.74)$ & 0.046 & -0.166 to 0.258 & 0.33 \\
\hline \multicolumn{8}{|c|}{$\begin{array}{l}n=\text { number of patients. } \\
\mathrm{B}_{1 \mathrm{j}}=\text { intervention effect: difference between groups in mean value of the outcome measure at } 3 \text { months corrected for baseline and for clustering and for } \\
\text { age and hours of appointment. } \\
\mathrm{Cl}=95 \% \text { confidence interval. } \\
{ }^{*} \text { Significant difference between intervention group and control group. }\end{array}$} \\
\hline
\end{tabular}


Table 5 Health symptoms and mastery: baseline and 12 months

\begin{tabular}{|c|c|c|c|c|c|c|c|}
\hline & \multicolumn{4}{|c|}{ Total $(n=117)$} & \multicolumn{3}{|c|}{ Intervention effect } \\
\hline & \multicolumn{2}{|c|}{ Intervention group ( $n=66$ ) } & \multicolumn{2}{|c|}{ Control group $(n=51)$} & \multicolumn{3}{|l|}{ Statistic } \\
\hline & Baseline & 12 months & Baseline & 12 months & $\mathrm{B}_{1 \mathrm{i}}$ & $\mathrm{Cl}$ & $\mathrm{p}$ \\
\hline \multicolumn{8}{|l|}{ Symptoms } \\
\hline \multicolumn{8}{|l|}{$4 \mathrm{DSQ}$} \\
\hline Distress, mean (SD) & $21.00(6.7)$ & $7.47(7.2)$ & $22.24(6.7)$ & $8.53(7.6)$ & 0.013 & -0.179 to 0.205 & 0.45 \\
\hline Depression, mean (SD) & $2.41(2.8)$ & $0.89(1.9)$ & $3.45(3.2)$ & $0.84(2.2)$ & -0.048 & -0.195 to 0.099 & 0.74 \\
\hline Anxiety, mean (SD) & $3.99(4.6)$ & $1.33(2.8)$ & $6.28(6.19)^{*}$ & $1.94(4.0)$ & -0.029 & -0.154 to 0.096 & 0.67 \\
\hline $\begin{array}{l}\text { Physical symptoms, mean (SD) } \\
\text { SCl-90 }\end{array}$ & $12.20(6.1)$ & $5.73(5.0)$ & $12.8(6.1)$ & $6.22(5.1)$ & 0.000 & -0.100 to 0.100 & 0.50 \\
\hline $\begin{array}{l}\text { SCL-90 } \\
\text { Total score, mean (SD) }\end{array}$ & $176(44)$ & $124(38)$ & $190(51)$ & $132(38)$ & -0.017 & -0.184 to 0.150 & 0.58 \\
\hline \multicolumn{8}{|l|}{$\begin{array}{l}\text { Psychological resources and } \\
\text { responses }\end{array}$} \\
\hline Mastery, mean (SD) & $3.22(0.66)$ & $3.42(0.95)$ & $3.18(0.69)$ & $3.54(0.77)$ & -0.112 & -0.414 to 0.190 & 0.77 \\
\hline $\begin{array}{l}n=\text { number of patients. } \\
\mathrm{B}_{1 \mathrm{i}}=\text { intervention effect: difference } \\
\text { age and hours of appointment. } \\
\mathrm{Cl}=95 \% \text { confidence interval. } \\
{ }^{*} \text { Significant difference between in }\end{array}$ & vention group & mean value o & outcome $\mathrm{m}$ & ure at $12 \mathrm{mo}$ & correcte & seline and for cluste & and for \\
\hline
\end{tabular}

months both groups had lower SCL-90 scores than a psychiatric outpatient group, but the scores were higher than the healthy reference group.

Almost all outcomes improved further in the period between 3 and 12 months, though less impressively than in the first 3 months. There were no significant treatment effects (see tables 4 and 5). For both groups at 12 months the SCL total score was still higher than the score for the healthy SCL reference population.

\section{DISCUSSION}

To our knowledge, the present study is the first controlled study evaluating the effectiveness of an intervention for adjustment disorders with occupational dysfunctioning. Clearly evidence based practice has yet to be introduced to this extensive field of mild but not always harmless disorders. The main aim of our intervention was to activate patients to develop and implement problem solving strategies for daily (working) life problems. The intervention was structured in a three stage approach based on a time contingency and graded activity approach. However, occupational physicians were free to draw up a guidance plan in consultation with the patient. The combination of a graded activity and a cognitivebehavioural problem solving approach in a structured but not protocolled intervention is a new approach.

We found the intervention to be effective on work ability at 3 months and on sickness leave duration, as hypothesised. This is consistent with the outcomes of graded activity programmes that report effects on work ability and sickness leave. ${ }^{8}{ }^{9}$ The size of the effects was comparable: a reduction of $25-30 \%$ in the duration of absenteeism compared with that of "care as usual". As "care as usual" in this specific situation was already rather effective, this should be interpreted as a treatment versus treatment result, more than as a treatment versus control result. Moreover, about $20 \%$ of the OPs in the control group recorded the use of specific tools that were part of the intervention method. The effectiveness of "care as usual" is also reflected by the finding that all patients, irrespective of their treatment group, had fully returned to work by 12 months. This is in sharp contrast with the outcome of the study by Schröer ${ }^{6}$ and underlines the favourable return to work rates of the company where the intervention took place. Results indicate that the intervention predominantly shortened long term absenteeism from about six weeks, as reflected in fig 1 . The difference in recurrence rate, though not significant in the occupational physician based analysis, suggests a lasting effect of the intervention. Both groups showed substantial improvements in symptoms and mastery, but there was no difference between the two groups.

Most interesting is that the study groups differ significantly in effectiveness regarding absenteeism related outcomes but not regarding symptom reduction. Apparently there is little correlation between symptoms and absenteeism. There are a number of possible interpretations.

Firstly, it could be stated that patients in the intervention group improved on symptoms as much as patients in the control group notwithstanding the fact that they were exposed to demanding activities and returned to work earlier. One could have expected that an earlier exposure to demanding activities would have an unfavourable effect on symptom reduction. Especially at the 3 months assessment there was a clear difference between both groups in return rates. Unfortunately, in our data we could not check the assumption that return to work would have a temporary negative effect on symptom improvement.

Secondly, the consistent change found in primary care patients between severity of psychiatric illness (depression and anxiety) and social disability ${ }^{28}$ might not count for the diagnosis "adjustment disorder" ${ }^{29}$ Thus, the more severe a psychiatric disorder the more improvement of psychiatric symptoms is needed before there is an effect at disability level. Adjustment disorders, defined more in terms of social dysfunctioning than in terms of symptoms, represent the concept of illness more than that of disease and can be regarded as a less severe health condition.

Another interpretation is that the graded activity component of our intervention was especially effective, leading to a faster building up of activities. That the cognitive component did not have the expected results on symptom reduction might be because this component was not distinguished from the cognitive elements of "care as usual", such as instruction. This is consistent with two British studies in which experimental interventions failed to have a significantly different effect from routine care. ${ }^{3}{ }^{30}$ In the Scott and Freeman study, ${ }^{30}$ one of the interventions was cognitive-behavioural therapy. It is not consistent with the findings of the meta-analysis of preventive interventions mentioned in the introduction. ${ }^{4}$ In this meta-analysis, cognitive-behavioural interventions had moderate effects on symptom levels and psychological resources and responses. However, only studies with a non-treatment control group were included in this meta-analysis.

Finally, the absence of a symptom effect may be due to the way we obtained our baseline data. The baseline questionnaire 
was given to the patients at the end of their first visit (intake). As company management had stipulated that the experiment should not cause any delay, treatment-either the intervention or care as usual-started at this first consultation. In addition, the importance of early treatment to reduce anxiety that could impair active coping by the patient was emphasised in the intervention protocol. As patients sometimes had to be reminded about the questionnaire at the second or even third consultation, there might have been some confounding by early treatment effects. In fact patients of the intervention group had more favourable baseline values on the 4DSQ, SCL 90 (especially on anxiety), and on the Mastery Scale compared with the control group.

We found some differences between completers and dropouts during the study but these differences counted equally for both study groups and did not seem to be a serious threat for the internal validity. Between the 3 months population and dropouts towards 12 months we found only one difference between the study groups indicating a potential selective dropout on this point. However, the interesting results of our study are focused on the absenteeism data, with "intention to treat" analyses on the whole study group without dropouts. These results are not threatened by the potential selectivity of dropout on self report measures between 3 and 12 months.

The single blind study design meant that bias could have been introduced because occupational physicians knew which treatment they provided. Occupational physicians in the intervention group might have had higher expectations of the quality of their guidance, and this could have influenced their effectiveness. We tried to avoid this by presenting the study as an evaluation of two equivalent strategies, the one being more structured than the other. Because the physicians were aware that the company had favourable sickness leave data compared with other companies, they knew that their "usual" care was adequate and they were proud of that. This way of presenting the study as an evaluation of two equivalent approaches was made more plausible by the initial reluctance of the company to approve the study, out of concern that the intervention might be less effective than usual care. There was awareness of the fact that psychological interventions are not always effective and are sometimes even harmful. ${ }^{31}$

Another limitation of our study was that random allocation on the patient level was not possible because this was unacceptable to the company. We randomly allocated occupational physicians to the intervention and the control group but this hierarchical design may introduce a selection bias. Subject selection can occur either when individuals are in the position to make a choice on the group level or as the group members share certain characteristics (which causes observations to be correlated within a group). An example of the first is as in a trial randomising medical practices, characteristics of patients could be related to, for example, sex or age differences among practitioners. An example of the second is as in a trial randomising families, family members share certain characteristics. The first phenomenon does not count in our study because there was no free choice of OP. With respect to the second phenomenon it is not plausible that there should be a strong relation among patients of an OP. There was a relation between the OP and the geographical area patients worked in, but those areas were quite extended and the area where patients lived was even larger. Moreover the areas OPs worked in overlapped. Sometimes there was a close work relation between the OP and the company division one worked in, but most OPs had many different divisions they worked for. Therefore, strong intraclass correlation caused by selection, threatening the internal validity of the study, is not probable. Nevertheless, we found some differences between the groups on baseline outcomes. We introduced these variables as covariates in our analyses in order to correct for possible bias.

Another potential drawback related to the hierarchical structure of our data, is that multilevel analyses, taking into account the variance on all levels, were not possible on the absenteeism data. This leaves the choice between two less perfect strategies: analyses on either the cluster level or the patient level. Because of the methodological rigour of experimental design which assumes that the experimental unit which is randomised is also the unit of analysis, ${ }^{32}$ we primarily analysed on the cluster level. This may have led to an underestimation of the significance of effects. As the point of inference in our study was so clearly on the individual level we also presented outcomes of analyses on the individual level, which might have led to an underestimation of $p$ values and an overestimation of the significance of effect as discussed in the methods section.

Because of an effective "care as usual", there was a relative lack of contrast between the control and intervention groups. It would be interesting to repeat the study in a situation with more contrast between the intervention and the control groups. Patient based randomisation and assessment of the baseline measures before the actual start of the intervention would correct for the imperfections in our study. Separate trials to evaluate the different parts of our intervention (that is, graded activity and a cognitive behavioural intervention) would provide more insight into the effectiveness of these components, apart and in interaction. It would be worthwhile to evaluate the effect of a more strict treatment protocol. It would also be valuable to test the intervention in other settings, for example, general practice and applied by other professionals. Indeed, an (uncontrolled) study with a more or less comparable intervention by general practitioners yielded favourable outcomes for work ability. ${ }^{33}$

We conclude that our intervention for adjustment disorders was successful in shortening sick leave duration, mainly by shortening long term absenteeism. This result is considered important not only because of the incidence of adjustment disorders but also because of the serious consequences they can have. Although symptoms may be self limiting, sickness leave can have a prolonged course and lead to permanent occupational disability and high work disability insurance costs. Our intervention appears to be successful in reducing the negative consequences of occupational dysfunctioning, and is as such a promising contribution to the prevention of chronic occupational disability caused by adjustment disorders. As the intervention was tested in a large company with a great variety of jobs, the results may be applicable to broad sections of the working population. We recommend the intervention as an option for the treatment of adjustment disorders with occupational dysfunctioning.

\section{ACKNOWLEDGEMENTS}

Grants were obtained from: The Occupational Health Service of Royal KPN; Netherlands Organisation of Scientific Research (NWO): Netherlands Concerted Research Action "Fatigue at Work"; TNO Work and Employment; and Foundation for Quality in Occupational Health (SKB). We thank Dr JWR Twisk and Prof. Dr GJ Bonsel for their advice on statistics.

n......

\section{Authors' affiliations}

J J L van der Klink, F J H van Dijk, Coronel Institute for Occupational and Environmental Health, Academic Medical Center, AmCOGG,

Amsterdam Center for Research in Health and Health Care, University of Amsterdam, Amsterdam, Netherlands

R W B Blonk, TNO Work and Employment

A H Schene, Department of Psychiatry, Academic Medical Center, AmCOGG, Amsterdam Center for Research in Health and Health Care, University of Amsterdam

\section{REFERENCES}

1 United Nations International Labor Organisation. Job stress: the 20th century disease. World Labor Report no. 6. Geneva, Switzerland: United Nations Labor Office, 1993.

2 Cartwright S, Cooper CL. Managing workplace stress. London: Sage Publications, 1997. 
3 Friedli K, King MB, Lloyd M, et al. Randomised controlled assessment of non-directive psychotherapy versus routine general-practitioner care. Lancet 1997;350:1662-5.

4 Klink JJL van der, RWB Blonk, AH Schene, et al. The benefits of interventions for work related stress. Am J Public Health 2001:91:270-6.

5 American Psychiatric Association. Diagnostic and statistical manual of mental disorders (fourth edition); DSM-IV. Washington, DC: American Psychiatric Press, 1994.

6 Schröer CAP. Absenteeism due to "overstrain". A study of the nature of overstrain, therapeutic assistance and absenteeism [in Dutch, with a summary in English]. Thesis. Maastricht: Universitaire Pers, 1993.

7 Eck MAA van. The diagnosing of mental disorders. In: Bijl R, Bauduin D, eds. Occupational disability due to mental disorders [in Dutch]. Utrecht: NCGV, 1991.

8 Fordyce WE. Back pain in the work place. Management of disability in nonspecific conditions: a report of the Task Force on Pain in the Workplace of the International Association for the Study of Pain. Seattle: IASP Press, 1995

9 Lindström I, Ohlund C, Eek C, et al. Mobility, strength, and fitness after a graded activity for patients with subacute low back pain. A randomized prospective clinical study with a behavioral therapy approach. Spine 1992;17:641-52.

10 Meichenbaum DH, Cameron R. Stress inoculation training. In: Meichenbaum DH, Jarenko ME, eds. Stress reduction and prevention. New York: Plenum Press, 1983.

11 Meichenbaum DH. Stress inoculation training: a twenty-year update. In: Woolfolk RL, Lehrer PM, eds. Principles and practice of stress management, 2nd edn. New York: Guilford Press, 1993

12 Saunders T, Driskell JE, Hall Johnston J, et al. The effect of stress inoculation training on anxiety and performance. J Occup Psychol 1997;70:170-86.

13 Fontana D. Managing stress, problems in practice. London: British Psychological Society, 1989

14 Nunnally JC. Psychometric theory. New York: McGraw Hill, 1978.

15 Gründemann RMW, Smulders PGW, Winter CR de. DWHQ, the Dutch Work and Health Questionnaire; Manual [in Dutch]. Lisse: Swets \& Zetlinger BV, 1993.

16 Schreurs PJG, Tellegen B, Willige G van de. Health, stress and coping: the development of the Utrecht Coping List [in Dutch]. Gedrag, Tijdschrift voor Psychologie 1984;12:101-17.

17 Schreurs PJG, Willige G van de, Brosschot JF, et al. De Utrecht Coping List: UCL; coping with problems and events; revised manual [in Dutch]. Lisse: Swets \& Zetlinger BV, 1993
18 Terluin B. The Four Dimensional Symptom Questionnaire (4DSQ); a questionnaire for assessing distress, depression, anxiety and somatisation [in Dutch]. Huisarts Wet 1996;39:538-47.

19 Terluin B. The Four Dimensional Symptom Questionnaire (4DSQ) in general practice. De Psycholoog 1998;33:18-24.

20 Derogatis LR. SCL-90: Administration, scoring and procedures manual-I for the (revised) version. Baltimore: Johns Hopkins University school of Medicine, Clinical Psychometrics Research Unit, 1977.

21 Derogatis LR, Cleary PA. Conformation of the dimensional structure of the SCL-90: a study in construct validity. J Clin Psychol 1977;33:981-9.

22 Arrindell WA, Ettema $\mathrm{H}$. Dimensional structure, reliability and validity of the Dutch version of the Symptom Checklist (SCL-90); data based on a phobic and a "normal" population [in Dutch]. Nederlands Tijdschrift voor de Psychologie 1981;36:77-108.

23 Arrindell WA, Ettema H. SCL-90, Manual of a multidimensional indicator of psychopathology [in Dutch]. Lisse: Swets \& Zeitlinger BV, 1986.

24 Pearlin LI, Schooler C. The structure of coping. J Health Soc Behav 1978;19:2-21.

25 Goldstein H. Multilevel statistical models. London: Arnold, 1995.

26 Goldstein H, Rashbash J, Plewis I, et al. A user's guide to MLwiN. London: Institute of Education, 1998.

27 Donner A, Klar N. Design and analysis of cluster randomization trials in health research. London: Arnold, 2000.

28 Ormel J, von Korff $M$, van den Brink W, et al. Depression, anxiety, and social disability show synchrony of change in primary care patients. Am J Public Health 1993:83:385-90.

29 Terluin B. Syndromes behind the diagnosis "overstrain"; a cluster analysis [in Dutch]. Huisarts Wet 1996;39:358-65.

30 Scott AIF, Freeman CPL. Edinburgh primary care depression study: treatment outcome, patient satisfaction, and costs after 16 weeks. BM 1992;304:883-7.

31 Rose S, Bisson J, Wessely S. Psychological debriefing for preventing post traumatic stress disorder (PTSD) (Cochrane review). In: The Cochrane Library, Issue 1. Oxford: Update Software, 2002.

32 Fischer RA. The design of experiments. Edinburgh: Oliver and Boyd, 1935.

33 Terluin B. Overstrain in general practice. The disbalance between mental workload and coping capacity [in Dutch]. Medisch Contact 1988;43: 1495-8. 